PUBLICATIONS DE L'INSTITUT MATHÉMATIQUE

Nouvelle série, tome 74(88) (2003), 85-95

\title{
A FUNCTION DEFINED ON AN EVEN-DIMENSIONAL REAL SUBMANIFOLD OF A HERMITIAN MANIFOLD
}

\author{
Masafumi Okumura
}

\author{
Communicated by Mileva Prvanović
}

\begin{abstract}
On an even-dimensional real submanifold of a Hermitian manifold, making use of the fundamental 2-form of the ambient manifold, we define a function. In this paper, we investigate the function in detail in some special submanifold.
\end{abstract}

\section{Introduction}

Let $M$ be an even-dimensional real submanifold of a Hermitian manifold $\bar{M}$. Then, making use of the fundamental 2-form of the ambient manifold, we can define a function $f$ on $M$. In [4], the present author and Y. Kubo defined the function and using this function, proved that an even-dimensional extrinsic sphere a of Kähler manifold is isometric with a sphere. Even though the final result in [4] is correct, there are some mistakes. In this paper, we correct these as well as investigate more properties of the function.

In Section 2 we recall some general preliminary facts on real submanifold of a Hermitian manifold and in Section 3 we define the function $f$ and give a concrete form of the function. In Section 4 we discuss the function on some kind of real submanifolds and show that in these cases it takes much simple form.

In Section 5 we consider the function on a totally umbilical submanifold and give a differential equation which the function should satisfy, from which we conclude that if the totally umbilical submanifold has parallel mean curvature vector field, the gradient of the function defines an infinitesimal concircular transformation. From this, together with the theorem of Obata $[\mathbf{2}]$, we prove that the submanifold $M$ is isometric with a sphere in Euclidean $(n+1)$-space. This is the correction of the paper [4]. Finally in Section 6 we consider the case that the totally umbilical submanifold is a submanifold of codimension 2 of complex submanifold and

2000 Mathematics Subject Classification: 53C15, 53C25, 53C40.

Key words and phrases: fundamental 2-form, totally umbilical submanifold, mean curvature vector field. 
give a concrete form of the second covariant derivative of the function which is a generalization of the result in $[\mathbf{3}]$.

The author wishes to thank the referee who read the manuscript carefully and pointed out the author's many careless mistakes.

\section{Even dimensional submanifold of a Hermitian manifold}

Let $\bar{M}$ be a real $(n+2 p)$-dimensional Hermitian manifold with Hermitian structure $(J, \bar{g})$, that is, $J$ is the almost complex structure of $\bar{M}$ and $\bar{g}$ the Riemannian metric of $\bar{M}$ satisfying the Hermitian condition $\bar{g}(J \bar{X}, J \bar{Y})=\bar{g}(\bar{X}, \bar{Y})$ for any $\bar{X}, \bar{Y} \in T(\bar{M})$. Let $M$ be an $n$-dimensional real submanifold of $\bar{M}$ and $\iota$ be the immersion. Then the tangent bundle $T(M)$ is identified with a subbundle of $T(\bar{M})$ and the induced Riemannian metric $g$ of $M$ is defined by $g(X, Y)=\bar{g}(\iota X, \iota Y)$ for $X, Y \in T(M)$, where we use the same $\iota$ for the differential map of the immersion $\iota$. The normal bundle $T^{\perp}(M)$ is the subbundle of $T(\bar{M})$ consisting of all $\bar{X} \in T(\bar{M})$ which are orthogonal to $T(M)$ with respect to $\bar{g}$. At each point of $M$, we choose orthonormal local vector fields $\xi_{1}, \ldots, \xi_{2 p}$ in such a way that they belong to $T^{\perp}(M)$. For any $X \in T(M)$ and for $\xi_{a}(a=1, \ldots, 2 p)$ the transforms $J \iota X$ and $J \xi_{a}$ are respectively written in the following forms:

$$
\begin{gathered}
J \iota X=\iota F X+\sum_{a=1}^{2 p} u^{a}(X) \xi_{a}, \\
J \xi_{a}=-\iota U_{a}+\sum_{b=1}^{2 p} p_{a b} \xi_{b},
\end{gathered}
$$

where $F, p_{a b}, U_{a}$ and $u^{a}$ define respectively an endomorphism of $T(M)$, that of $T^{\perp}(M)$, local tangent vector fields and local 1-forms on $M$. They satisfy the relations $u^{a}(X)=g\left(U_{a}, X\right)$ and $p_{a b}=-p_{b a}$. If $U_{a}, a=1, \ldots, 2 p$ vanish identically, the tangent space of $M$ is invariant under $J$ and in this case the submanifold is a complex manifold with induced almost complex structure.

Applying $J$ to both side members of (2.1) and (2.2), we find

$$
\begin{gathered}
F^{2} X=-X+\sum_{a=1}^{2 p} u^{a}(X) U_{a}, \\
u^{a}(F X)=-\sum_{b=1}^{2 p} p_{b a} u^{b}(X), \quad F U_{a}=-\sum_{b=1}^{2 p} p_{a b} U_{b}, \quad a=1, \ldots, 2 p, \\
\sum_{c=1}^{2 p} p_{a c} p_{c b}=-\delta_{a b}+u^{b}\left(U_{a}\right), \quad a, b=1, \ldots, 2 p .
\end{gathered}
$$

We denote by $\bar{\nabla}$ and $\nabla$ the Riemannian connection of $\bar{M}$ and $M$ respectively and by $D$ the induced normal connection from $\bar{\nabla}$ to $T^{\perp}(M)$. Then they are related by the following equations [1]: 


$$
\begin{gathered}
\bar{\nabla}_{\iota X} \iota Y=\iota \nabla_{X} Y+h(X, Y) \\
\bar{\nabla}_{\iota X} \xi_{a}=-\iota A_{a} X+D_{X} \xi_{a}, \quad D_{X} \xi_{a}=\sum_{b=1}^{2 p} s_{a b}(X) \xi_{b}, \quad a=1, \ldots, 2 p
\end{gathered}
$$

where $h$ is the second fundamental form and $A_{a}$ is a symmetric linear transformation of $T(M)$, which is called the shape operator with respect to $\xi_{a}$. The last two equations show that $h(X, Y)=\sum_{a=1}^{2 p} g\left(A_{a} X, Y\right) \xi_{a}$.

The mean curvature vector field $\mu$ of $M$ is defined by

$$
\mu=\frac{1}{n} \sum_{a=1}^{2 p}\left(\operatorname{trace} A_{a}\right) \xi_{a},
$$

and it is well-known that $\mu$ is independent of the choice of orthonormal normals $\xi_{1}, \ldots, \xi_{2 p}$. The length of the mean curvature vector field is called the mean curvature of the submanifold and it is given by

$$
|\mu|=\frac{1}{n}\left\{\sum_{a=1}^{2 p}\left(\operatorname{trace} A_{a}\right)^{2}\right\}^{1 / 2} .
$$

Differentiating (2.8) covariantly, we get

$$
n D_{X} \mu=\sum_{a=1}^{2 p}\left\{X\left(\operatorname{trace} A_{a}\right) \xi_{a}+\sum_{b=1}^{2 p}\left(\operatorname{trace} A_{a}\right) s_{a b}(X) \xi_{b}\right\},
$$

from which we know that the mean curvature vector field is parallel with respect to the normal connection if and only if

$$
X\left(\operatorname{trace} A_{a}\right)=\sum_{b=1}^{2 p}\left(\operatorname{trace} A_{b}\right) s_{a b}(X),
$$

because of the fact that $s_{a b}=-s_{b a}$.

\section{A function defined by the fundamental 2-form of $\bar{M}$}

Let $\Omega$ be the fundamental 2 -form of the Hermitian manifold $\bar{M}$, that is, $\Omega$ is defined by

$$
\Omega(\bar{X}, \bar{Y})=\bar{g}(J \bar{X}, \bar{Y})
$$

for $\bar{X}, \bar{Y} \in T(\bar{M})$. We put

$$
f=\Omega^{p}\left(\xi_{1}, \xi_{2}, \ldots, \xi_{2 p}\right),
$$

where $\Omega^{p}$ denotes $p$-times exterior product of $\Omega$. Then we have

LEMma 3.1. $f$ is independent of the choice of mutually orthonormal normals $\xi_{1}, \ldots, \xi_{2 p}$. 
Proof. Let $\eta_{1}, \ldots, \eta_{2 p}$ be another choice of mutually orthonormal normals to $M$. Then we have

$$
\eta_{a}=\sum_{b=1}^{2 p} T_{a}^{b} \xi_{b}
$$

for some orthogonal matrix $\left(T_{a}^{b}\right) \in O(2 p)$. Denoting by $S(2 p)$ the symmetric group of order $2 p$, we have from $(3.2)$

$$
\begin{aligned}
f^{\prime}=\Omega^{p}\left(\eta_{1}, \ldots, \eta_{2 p}\right) & =\sum_{c_{1}, \ldots, c_{2 p}} T_{1}^{c_{1}} T_{2}^{c_{2}} \cdots T_{2 p}^{c_{2 p}} \Omega^{p}\left(\xi_{c_{1}}, \xi_{c_{2}}, \ldots, \xi_{c_{2 p}}\right) \\
& =\sum_{\sigma \in S(2 p)} T_{1}^{\sigma(1)} T_{2}^{\sigma(2)} \cdots T_{2 p}^{\sigma(2 p)} \Omega^{p}\left(\xi_{\sigma(1)}, \xi_{\sigma(2)}, \ldots, \xi_{\sigma(2 p)}\right) \\
& =\sum_{\sigma \in S(2 p)} T_{1}^{\sigma(1)} T_{2}^{\sigma(2)} \cdots T_{2 p}^{\sigma(2 p)} \operatorname{sgn} \sigma \Omega^{p}\left(\xi_{1}, \xi_{2}, \ldots, \xi_{2 p}\right) \\
& =\operatorname{det}\left(T_{a}^{b}\right) \Omega^{p}\left(\xi_{1}, \ldots, \xi_{2 p}\right)=f .
\end{aligned}
$$

This shows that $f$ is independent of the choice of normals.

Now we discuss the function $f$ more concretely. The number $A$ of the terms in the expansion of $f$ as a sum of product $\Omega\left(\xi_{a_{1}}, \xi_{a_{2}}\right) \Omega\left(\xi_{a_{3}}, \xi_{a_{4}}\right) \cdots \Omega\left(\xi_{a_{2 p-1}}, \xi_{a_{2 p}}\right)$ is

$$
\left(\begin{array}{c}
2 p \\
2
\end{array}\right)\left(\begin{array}{c}
2 p-2 \\
2
\end{array}\right) \cdots\left(\begin{array}{l}
4 \\
2
\end{array}\right)\left(\begin{array}{l}
2 \\
2
\end{array}\right)=\frac{(2 p) !}{2^{p}},
$$

and the number $B$ of different factorization of $\Omega\left(\xi_{a_{i}}, \xi_{a_{j}}\right)$ 's is $(2 p-1)(2 p-3) \cdots 5 \cdot 3 \cdot 1$. Hence, in the expansion, there are $A / B=p$ ! like terms. Hence we can write

$$
f=p ! \sum \Omega\left(\xi_{a_{1}}, \xi_{a_{2}}\right) \cdots \Omega\left(\xi_{a_{2 p-1}}, \xi_{a_{2 p}}\right),
$$

where $\sum$ means the sum of all such combinations of $a_{2 l-1}, a_{2 l} \in\{1,2, \ldots, 2 p\}$ that $a_{2 l-1}<a_{2 l}$.

\section{Complex submanifolds, CR submanifolds of CR dimension $\frac{n-2}{2}$}

Let $M$ be a complex submanifold of a Hermitian manifold $\bar{M}$. Since the normal space $T_{x}^{\perp}(M)$ of $x \in M$ is $J$-invariant subspace of $T_{x}(\bar{M})$ as well as the tangent space $T_{x}(M)$, we can choose an orthonormal basis of $T_{x}^{\perp}(M)$ in such a way that $\left(\xi_{2 a}\right)_{x}=J\left(\xi_{2 a-1}\right)_{x}, a=1, \ldots, p$ and extend them to local fields $\xi_{1}, \ldots, \xi_{2 p}$. Then we have

$$
\begin{gathered}
\Omega\left(\xi_{2 a-1}, \xi_{2 b-1}\right)=\bar{g}\left(J \xi_{2 a-1}, \xi_{2 b-1}\right)=\bar{g}\left(\xi_{2 a}, \xi_{2 b-1}\right)=0 \\
\Omega\left(\xi_{2 a-1}, \xi_{2 b}\right)=\bar{g}\left(J \xi_{2 a-1}, \xi_{2 b}\right)=\bar{g}\left(\xi_{2 a}, \xi_{2 b}\right)=\delta_{a b} .
\end{gathered}
$$

Thus in (3.3), $\Omega\left(\xi_{a_{1}}, \xi_{a_{2}}\right) \cdots \Omega\left(\xi_{a_{2 p-1}}, \xi_{a_{2 p}}\right)=0$, except

$$
\Omega\left(\xi_{1}, \xi_{2}\right) \Omega\left(\xi_{3}, \xi_{4}\right) \cdots \Omega\left(\xi_{2 p-1}, \xi_{2 p}\right)=1 .
$$

Hence, from (3.3) $f=p$ !. Thus we have

Proposition 4.1. For a complex submanifold $M$ we have $f=p !$. 
Now we consider a CR submanifold $M$ of CR dimension $(n-2) / 2$ in a Hermitian manifold $M$. By definition, at each point $x \in M$, the real dimension of the holomorphic tangent space $H_{x}(M)=J T_{x}(M) \cap T_{x}(M)$ is $n-2$. We choose an orthonormal basis $e_{1}, e_{2}, \ldots, e_{n}$ of $T_{x}(M)$ in such a way that $e_{1}, e_{2}, \ldots, e_{n-2} \in H_{x}(M)$. Then $J \iota e_{j} \in T_{x}(M),(j=1, \ldots, n-2)$ and

$$
J \iota e_{n-1}=\lambda \iota e_{n}+\eta_{1}, \quad J \iota e_{n}=-\lambda \iota e_{n-1}+\eta_{2},
$$

where $\eta_{1}$ and $\eta_{2}$ denote the normal part of $J \iota e_{n-1}$ and $J \iota e_{n}$ respectively. We note that $\eta_{1}$ and $\eta_{2}$ never vanish. In fact, if, for example, $\eta_{1}$ vanishes at a point $x \in M$, from the first equation of (4.1) it follows that $J \iota e_{n-1} \in T_{x}(M)$. This shows that the real dimension of $H_{x}(M)$ at $x$ is greater than $n-2$. This is a contradiction.

We choose orthonormal normal vectors $\xi_{1}, \xi_{2}, \ldots, \xi_{2 p}$ to $M$ in such a way that $\xi_{1}$ and $\xi_{2}$ are in the direction of $\eta_{1}$ and $\eta_{2}$ respectively, that is, $\xi_{1}=\eta_{1} /\left|\eta_{1}\right|$, $\xi_{2}=\eta_{2} /\left|\eta_{2}\right|$. Then we have for $a=3, \ldots, 2 p$,

$$
\begin{gathered}
\bar{g}\left(J \xi_{a}, \iota X\right)=-\bar{g}\left(\xi_{a}, J \iota X\right)=0, \\
\bar{g}\left(J \xi_{a}, \xi_{1}\right)=-\bar{g}\left(\xi_{a}, J \xi_{1}\right)=0, \\
\bar{g}\left(J \xi_{a}, \xi_{2}\right)=-\bar{g}\left(\xi_{a}, J \xi_{2}\right)=0,
\end{gathered}
$$

because of (4.1). These equations show that the subspace $\operatorname{span}\left\{\xi_{3}, \ldots, \xi_{2 p}\right\}$ of $T^{\perp}(M)$ is $J$-invariant and therefore we choose such an orthonormal basis of $\operatorname{span}\left\{\xi_{3}\right.$, $\left.\ldots, \xi_{2 p}\right\}$ that $\xi_{2 a}=J \xi_{2 a-1}, a=2, \ldots, p$. By choosing these orthonormal normals, it follows that, for $a, b \geqslant 2$ :

$$
\begin{aligned}
\Omega\left(\xi_{2 a-1}, \xi_{2 b-1}\right) & =\bar{g}\left(J \xi_{2 a-1}, \xi_{2 b-1}\right)=\bar{g}\left(\xi_{2 a}, \xi_{2 b-1}\right)=0, \\
\Omega\left(\xi_{2 a-1}, \xi_{2 b}\right) & =\bar{g}\left(J \xi_{2 a-1}, \xi_{2 b}\right)=\bar{g}\left(\xi_{2 a}, \xi_{2 b}\right)=\delta_{a b},
\end{aligned}
$$

and for $a \geqslant 3$ :

$$
\Omega\left(\xi_{1}, \xi_{a}\right)=\bar{g}\left(J \xi_{1}, \xi_{a}\right)=0, \quad \Omega\left(\xi_{2}, \xi_{a}\right)=\bar{g}\left(J \xi_{2}, \xi_{a}\right)=0 .
$$

Hence in (3.3), only the term $\Omega\left(\xi_{1}, \xi_{2}\right) \Omega\left(\xi_{3}, \xi_{4}\right) \cdots \Omega\left(\xi_{2 p-1}, \xi_{2 p}\right)$ does not vanish and consequently we have

Proposition 4.2. For a $C R$ submanifold $M$ of $C R$ dimension $(n-2) / 2$ we have $f=p ! \Omega\left(\xi_{1}, \xi_{2}\right)$.

Next let $M$ be a real submanifold of codimension 2 of a complex submanifold $M^{\prime}$ of a Hermitian manifold $\bar{M}$. We choose orthonormal normals to $M$ in $\bar{M}$ in such a way that $\xi_{1}, \xi_{2} \in T\left(M^{\prime}\right)$ and $\xi_{3}, \ldots, \xi_{2 p} \in T^{\perp}\left(M^{\prime}\right)$. As $M^{\prime}$ is a complex submanifold, $T^{\perp}\left(M^{\prime}\right)$ is $J$-invariant. Hence, in entirely the same argument as in the case that $M$ is CR submanifold of CR dimension $(n-2) / 2$, we have

Proposition 4.3. For a real submanifold of codimension 2 of a complex submanifold of a Hermitian manifold we have $f=p ! \Omega\left(\xi_{1}, \xi_{2}\right)$. 


\section{Totally umbilical submanifold of a Kähler manifold}

Let $M$ be a submanifold of $\bar{M}$. If at each point of $M$ there exist differentiable functions $\rho_{a}, a=1,2, \ldots, 2 p$, satisfying

$$
A_{a} X=\rho_{a} X
$$

for any $X \in T(M)$, we call the submanifold a totally umbilical submanifold. In this case $\rho_{a}=\left(\operatorname{trace} A_{a}\right) / n$, that is,

$$
A_{a} X=\frac{1}{n}\left(\operatorname{trace} A_{a}\right) X
$$

First we consider the function $f$ on a typical example of a totally umbilical submanifold.

Example 1. Let $\bar{M}$ be a complex space $\mathbf{C}^{(n+2) / 2}$ with complex coordinates $z^{\lambda}=x^{\lambda}+\sqrt{-1} y^{\lambda},(\lambda=1, \ldots, q=(n+2) / 2)$. An $n$-dimensional sphere $S^{n}$ defined by

$$
S^{n}=\left\{\left(x^{\lambda}, y^{\lambda}\right) \mid \sum_{\lambda=1}^{q}\left\{\left(x^{\lambda}\right)^{2}+\left(y^{\lambda}\right)^{2}\right\}=1, y^{q}=0\right\}
$$

is a totally umbilical submanifold of codimension 2 of $\mathbf{C}^{(n+2) / 2}$. We choose mutually orthonormal vector fields $\xi_{1}$ and $\xi_{2}$ to $S^{n}$ as

$$
\xi_{1}=\sum_{\lambda=1}^{q}\left(x^{\lambda} \frac{\partial}{\partial x^{\lambda}}+y^{\lambda} \frac{\partial}{\partial y^{\lambda}}\right), \quad \xi_{2}=\frac{\partial}{\partial y^{q}} .
$$

Then $J \xi_{2}=-\partial / \partial x^{q}$. Since codimension is 2 , the function $f=\Omega\left(\xi_{1}, \xi_{2}\right)=$ $-\bar{g}\left(\xi_{1}, J \xi_{2}\right)=x^{q}$. Thus, in this case, $f$ is the level function of the last real coordinate.

From now on we assume that the ambient manifold $\bar{M}$ is a Kähler manifold. Then $J$ is covariant constant and therefore $\nabla_{X} \Omega=0$. So, from (2.7):

$$
\begin{aligned}
g(\operatorname{grad} f, Y) & =Y\left(\Omega^{p}\left(\xi_{1}, \ldots, \xi_{2 p}\right)\right)=\sum_{a=1}^{2 p} \Omega^{p}\left(\xi_{1}, \ldots, \xi_{a-1}, \bar{\nabla}_{\iota Y} \xi_{a}, \xi_{a+1}, \ldots, \xi_{2 p}\right) \\
& =\sum_{a=1}^{2 p} \Omega^{p}\left(\xi_{1}, \ldots, \xi_{a-1},-\iota A_{a} Y+\sum_{b=1}^{2 p} s_{a b}(Y) \xi_{b}, \xi_{a+1}, \ldots, \xi_{2 p}\right) \\
& =-\sum_{a=1}^{2 p} \Omega^{p}\left(\xi_{1}, \ldots, \xi_{a-1}, \iota A_{a} Y, \xi_{a+1}, \ldots, \xi_{2 p}\right),
\end{aligned}
$$

because $\Omega^{p}$ is a $2 p$-form and $s_{a b}$ are skew-symmetric with respect to $a$ and $b$. Then it follows that

$$
\begin{aligned}
g\left(\nabla_{X} \operatorname{grad} f, Y\right) & =X(g(\operatorname{grad} f, Y))-g\left(\operatorname{grad} f, \nabla_{X} Y\right) \\
& =-X\left(\sum_{a=1}^{2 p} \Omega^{p}\left(\xi_{1}, \ldots, \xi_{a-1}, \iota A_{a} Y, \xi_{a+1}, \ldots, \xi_{2 p}\right)\right)-g\left(\operatorname{grad} f, \nabla_{X} Y\right)
\end{aligned}
$$




$$
\begin{aligned}
= & -\sum_{b=1}^{a-1} \sum_{a=1}^{2 p} \Omega^{p}\left(\xi_{1}, \ldots, \bar{\nabla}_{\iota X} \xi_{b}, \ldots, \xi_{a-1}, \iota A_{a} Y, \xi_{a+1}, \ldots, \xi_{2 p}\right) \\
& -\sum_{a=1}^{2 p} \Omega^{p}\left(\xi_{1}, \ldots, \xi_{a-1}, \bar{\nabla}_{\iota X} \iota A_{a} Y, \xi_{a+1}, \ldots, \xi_{2 p}\right) \\
& -\sum_{b=a+1}^{2 p} \sum_{a=1}^{2 p} \Omega^{p}\left(\xi_{1}, \ldots, \xi_{a-1}, \iota A_{a} Y, \xi_{a+1}, \ldots, \bar{\nabla}_{\iota X} \xi_{b}, \ldots, \xi_{2 p}\right) \\
& -g\left(\operatorname{grad} f, \nabla_{X} Y\right) .
\end{aligned}
$$

Substituting (2.7) into the above equation and making use of the fact that

$$
g\left(\operatorname{grad} f, \nabla_{X} Y\right)=-\sum_{a=1}^{2 p} \Omega^{p}\left(\xi_{1}, \ldots, \xi_{a-1}, \iota A_{a} \nabla_{X} Y, \xi_{a+1}, \ldots, \xi_{2 p}\right),
$$

we have

$$
\begin{aligned}
& g\left(\nabla_{X} \operatorname{grad} f, Y\right)=\sum_{b=1}^{a-1} \sum_{a=1}^{2 p} \Omega^{p}\left(\xi_{1}, \ldots, \iota A_{b} X, \ldots, \xi_{a-1}, \iota A_{a} Y, \xi_{a+1}, \ldots, \xi_{2 p}\right) \\
& -\sum_{b=1}^{a-1} \sum_{a, c=1}^{2 p} s_{b c}(X) \Omega^{p}\left(\xi_{1}, \ldots, \xi_{b-1}, \xi_{c}, \xi_{b+1}, \ldots, \iota A_{a} Y, \ldots, \xi_{2 p}\right) \\
& -\sum_{a=1}^{2 p} \Omega^{p}\left(\xi_{1}, \ldots, \xi_{a-1}, \iota\left(\nabla_{X} A_{a}\right) Y, \xi_{a+1}, \ldots, \xi_{2 p}\right) \\
& -\sum_{a, b=1}^{2 p} g\left(A_{b} A_{a} Y, X\right) \Omega^{p}\left(\xi_{1}, \ldots, \xi_{a-1}, \xi_{b}, \xi_{a+1}, \ldots, \xi_{2 p}\right) \\
& +\sum_{b=a+1}^{2 p} \sum_{a=1}^{2 p} \Omega^{p}\left(\xi_{1}, \ldots, \xi_{a-1}, \iota A_{a} Y, \xi_{a+1}, \ldots, \iota A_{b} X, \ldots, \xi_{2 p}\right) \\
& -\sum_{b=a+1}^{2 p} \sum_{a, c=1}^{2 p} s_{b c}(X) \Omega^{p}\left(\xi_{1}, \ldots, \xi_{a-1}, \iota A_{a} Y, \ldots, \xi_{b-1}, \xi_{c}, \xi_{b+1}, \ldots, \xi_{2 p}\right) \\
& =\sum_{b=1}^{a-1} \sum_{a=1}^{2 p} \Omega^{p}\left(\xi_{1}, \ldots, \iota A_{b} X, \ldots, \xi_{a-1}, \iota A_{a} Y, \xi_{a+1}, \ldots, \xi_{2 p}\right) \\
& -\sum_{b=1}^{a-1} \sum_{a=1}^{2 p} s_{b a}(X) \Omega^{p}\left(\xi_{1}, \ldots, \xi_{b-1}, \xi_{a}, \xi_{b+1}, \ldots, \iota A_{a} Y, \ldots, \xi_{2 p}\right) \\
& -\sum_{a=1}^{2 p} \Omega^{p}\left(\xi_{1}, \ldots, \xi_{a-1}, \iota\left(\nabla_{X} A_{a}\right) Y, \xi_{a+1}, \ldots, \xi_{2 p}\right)-\sum_{a=1}^{2 p} g\left(A_{a}^{2} Y, X\right) f \\
& +\sum_{b=a+1}^{2 p} \sum_{a=1}^{2 p} \Omega^{p}\left(\xi_{1}, \ldots, \xi_{a-1}, \iota A_{a} Y, \xi_{a+1}, \ldots, \iota A_{b} X, \ldots, \xi_{2 p}\right)
\end{aligned}
$$




$$
\begin{aligned}
& -\sum_{b=a+1}^{2 p} \sum_{a=1}^{2 p} s_{b a}(X) \Omega^{p}\left(\xi_{1}, \ldots, \iota A_{a} Y, \ldots, \xi_{b-1}, \xi_{a}, \xi_{b+1}, \ldots, \xi_{2 p}\right) \\
= & \sum_{b=1}^{a-1} \sum_{a=1}^{2 p} \Omega^{p}\left(\xi_{1}, \ldots, \iota A_{b} X, \ldots, \xi_{a-1}, \iota A_{a} Y, \xi_{a+1}, \ldots, \xi_{2 p}\right) \\
& +\sum_{b=1}^{a-1} \sum_{a=1}^{2 p} s_{b a}(X) \Omega^{p}\left(\xi_{1}, \ldots, \xi_{b-1}, \iota A_{a} Y, \xi_{b+1}, \ldots, \xi_{a}, \ldots, \xi_{2 p}\right) \\
& -\sum_{a=1}^{2 p} \Omega^{p}\left(\xi_{1}, \ldots, \xi_{a-1}, \iota\left(\nabla_{X} A_{a}\right) Y, \xi_{a+1}, \ldots, \xi_{2 p}\right)-\sum_{a=1}^{2 p} g\left(A_{a}^{2} Y, X\right) f \\
& +\sum_{b=a+1}^{2 p} \sum_{a=1}^{2 p} \Omega^{p}\left(\xi_{1}, \ldots, \xi_{a-1}, \iota A_{a} Y, \xi_{a+1}, \ldots, \iota A_{b} X, \ldots, \xi_{2 p}\right) \\
& +\sum_{b=a+1}^{2 p} \sum_{a=1}^{2 p} s_{b a}(X) \Omega^{p}\left(\xi_{1}, \ldots, \xi_{a}, \ldots, \xi_{b-1}, \iota A_{a} Y, \xi_{b+1}, \ldots, \xi_{2 p}\right) .
\end{aligned}
$$

If $M$ is a totally umbilical submanifold, by (5.1), it follows that

$$
g(\operatorname{grad} f, Y)=-\sum_{a=1}^{2 p} \rho_{a} \Omega^{p}\left(\xi_{1}, \ldots, \xi_{a-1}, \iota Y, \xi_{a+1}, \ldots, \xi_{2 p}\right)
$$

and

$$
\begin{aligned}
g\left(\nabla_{X} \operatorname{grad} f, Y\right)= & \sum_{b=1}^{a-1} \sum_{a=1}^{2 p} \rho_{a} \rho_{b} \Omega^{p}\left(\xi_{1}, \ldots, \iota X, \ldots, \xi_{a-1}, \iota Y, \xi_{a+1}, \ldots, \xi_{2 p}\right) \\
& +\sum_{b=1}^{a-1} \sum_{a=1}^{2 p} \rho_{a} s_{b a}(X) \Omega^{p}\left(\xi_{1}, \ldots, \xi_{b-1}, \iota Y, \xi_{b+1}, \ldots, \xi_{2 p}\right) \\
& -\sum_{a=1}^{2 p}\left(X \rho_{a}\right) \Omega^{p}\left(\xi_{1}, \ldots, \xi_{a-1}, \iota Y, \xi_{a+1}, \ldots, \xi_{2 p}\right)-\left(\sum_{a=1}^{2 p} \rho_{a}^{2}\right) g(X, Y) f \\
& +\sum_{b=a+1}^{2 p} \sum_{a=1}^{2 p} \rho_{a} \rho_{b} \Omega^{p}\left(\xi_{1}, \ldots, \xi_{a-1}, \iota Y, \xi_{a+1}, \ldots, \iota X, \ldots, \xi_{2 p}\right) \\
& +\sum_{b=a+1}^{2 p} \sum_{a=1}^{2 p} \rho_{a} s_{b a}(X) \Omega^{p}\left(\xi_{1}, \ldots, \xi_{b-1}, \iota Y, \xi_{b+1}, \ldots, \xi_{2 p}\right) \\
= & -\sum_{a=1}^{2 p}\left(X \rho_{a}\right) \Omega^{p}\left(\xi_{1}, \ldots, \xi_{a-1}, \iota Y, \xi_{a+1}, \ldots, \xi_{2 p}\right)-\left(\sum_{a=1}^{2 p} \rho_{a}^{2}\right) f g(X, Y) \\
& +\sum_{a=1}^{b-1} \sum_{b=1}^{2 p} \rho_{b} s_{a b}(X) \Omega^{p}\left(\xi_{1}, \ldots, \xi_{a-1}, \iota Y, \xi_{a+1}, \ldots, \xi_{2 p}\right)
\end{aligned}
$$




$$
\begin{aligned}
& +\sum_{a=b+1}^{2 p} \sum_{b=1}^{2 p} \rho_{b} s_{a b}(X) \Omega^{p}\left(\xi_{1}, \ldots, \xi_{a-1}, \iota Y, \xi_{a+1}, \ldots, \xi_{2 p}\right) \\
= & -\sum_{a=1}^{2 p}\left(X \rho_{a}\right) \Omega^{p}\left(\xi_{1}, \ldots, \xi_{a-1}, \iota Y, \xi_{a+1}, \ldots, \xi_{2 p}\right)-\left(\sum_{a=1}^{2 p} \rho_{a}^{2}\right) f g(X, Y) \\
& +\sum_{a=1}^{2 p} \sum_{b=1}^{2 p} \rho_{b} s_{a b}(X) \Omega^{p}\left(\xi_{1}, \ldots, \xi_{a-1}, \iota Y, \xi_{a+1}, \ldots, \xi_{2 p}\right),
\end{aligned}
$$

because of $s_{b b}=0$. Thus we have

$$
\begin{aligned}
g\left(\nabla_{X} \operatorname{grad} f, Y\right)= & -\left(\sum_{a=1}^{2 p} \rho_{a}^{2}\right) f g(X, Y) \\
& -\sum_{a=1}^{2 p}\left(X \rho_{a}-\sum_{b=1}^{2 p} \rho_{b} s_{a b}(X)\right) \Omega^{p}\left(\xi_{1}, \ldots, \xi_{a-1}, \iota Y, \xi_{a+1}, \ldots, \xi_{2 p}\right)
\end{aligned}
$$

Hence we obtain

THEOREM 5.1. In an even dimensional totally umbilical submanifold $M$ of a Kähler manifold, the function $f$ satisfies (5.5). Moreover, if the mean curvature vector field is parallel with respect to the normal connection, $f$ satisfies

$$
g\left(\nabla_{X} \operatorname{grad} f, Y\right)=-\left(\sum_{a=1}^{2 p} \rho_{a}^{2}\right) f g(X, Y) .
$$

In connection with the function which satisfies (5.6), Obata $[\mathbf{2}]$ proved that in an $n$-dimensional complete, connected Riemannian manifold $M$, if there exists a function $f$ satisfying

$$
g\left(\nabla_{X} \operatorname{grad} f, Y\right)=-k^{2} f g(X, Y),
$$

for some constant $k, M$ is isometric with a sphere of radius $1 / k$ in the Euclidean $(n+1)$-space. Further, it can be easily proved that if the mean curvature vector field is parallel with respect to the normal connection, then $|\mu|$ is constant and since $\sum_{a=1}^{2 p} \rho_{a}^{2}=|\mu|^{2}$, it follows:

THEOREM 5.2. [4] Let $M$ be an even dimensional complete, connected totally umbilical submanifold of a Kähler manifold. If the mean curvature vector field is parallel with respect to the normal connection, then $M$ is isometric with a sphere of radius $1 /|\mu|$.

\section{Totally umbilical submanifold which is a submanifold of codimension 2 of a complex submanifold}

Here, let $M$ be a submanifold of codimension 2 of a complex submanifold $M^{\prime}$ of a Kähler manifold $\bar{M}$. Then, in entirely the same argument which we used to get Proposition 4.3, it follows that

$$
\Omega^{p}\left(\iota Y, \xi_{2}, \xi_{3}, \ldots, \xi_{2 p}\right)=p ! \Omega\left(\iota Y, \xi_{2}\right), \quad \Omega^{p}\left(\xi_{1}, \iota Y, \xi_{3}, \ldots, \xi_{2 p}\right)=p ! \Omega\left(\xi_{1}, \iota Y\right)
$$




$$
\Omega^{p}\left(\xi_{1}, \xi_{2}, . ., \xi_{a-1}, \iota Y, \xi_{a+1}, \ldots, \xi_{2 p}\right)=0,(a=3, \ldots, 2 p),
$$

where we have chosen orthonormal normals $\xi_{1}, \ldots, \xi_{2 p}$ to $M$ in such a way that $\xi_{1}, \xi_{2} \in T\left(M^{\prime}\right)$ and $\xi_{3}, \ldots, \xi_{2 p}$ are normal to $M^{\prime}$.

Since the tangent space of a complex submanifold $M^{\prime}$ is $J$-invariant, for $\xi_{1}$ and $\xi_{2}$, we have

$$
\begin{gathered}
J \xi_{1}=-\iota U_{1}+\lambda \xi_{2}, \quad J \xi_{2}=-\iota U_{2}-\lambda \xi_{1}, \quad \lambda=\Omega\left(\xi_{1}, \xi_{2}\right), \\
g\left(U_{1}, U_{1}\right)=g\left(U_{2}, U_{2}\right)=1-\lambda^{2}, g\left(U_{1}, U_{2}\right)=0, U_{a}=0(a=3, \ldots, 2 p), \\
J \iota X=\iota F X+u^{1}(X) \xi_{1}+u^{2}(X) \xi_{2}, \\
\Omega\left(\iota Y, \xi_{2}\right)=g\left(U_{2}, Y\right), \Omega\left(\xi_{1}, \iota Y\right)=-g\left(U_{1}, Y\right) .
\end{gathered}
$$

Now we assume that $M$ is totally umbilical in $\bar{M}$. Then, from (6.1), (6.2) and (6.6), (5.5) becomes

(6.7) $g\left(\nabla_{X} \operatorname{grad} f, Y\right)=-|\mu|^{2} f g(X, Y)-p !\left\{\left(D_{X} \rho_{1}\right) g\left(U_{2}, Y\right)-\left(D_{X} \rho_{2}\right) g\left(U_{1}, Y\right)\right\}$

where $D_{X} \rho_{1}=X \rho_{1}-\rho_{2} s_{12}(X)$ and $D_{X} \rho_{2}=X \rho_{2}-\rho_{1} s_{21}(X)$. Since the left-hand member of (6.7) is symmetric with respect to $X, Y$, it follows that

$$
\left(D_{X} \rho_{1}\right) g\left(U_{2}, Y\right)-\left(D_{X} \rho_{2}\right) g\left(U_{1}, Y\right)=\left(D_{Y} \rho_{1}\right) g\left(U_{2}, X\right)-\left(D_{Y} \rho_{2}\right) g\left(U_{1}, X\right) .
$$

Substituting $Y$ in the last equation for $U_{2}$ and making use of (6.4), we have

$$
\left(1-\lambda^{2}\right) D_{X} \rho_{1}=\left(D_{U_{2}} \rho_{1}\right) g\left(U_{2}, X\right)-\left(D_{U_{2}} \rho_{2}\right) g\left(U_{1}, X\right)
$$

Similarly, we have

$$
\left(1-\lambda^{2}\right) D_{X} \rho_{2}=\left(D_{U_{1}} \rho_{2}\right) g\left(U_{1}, X\right)-\left(D_{U_{1}} \rho_{1}\right) g\left(U_{2}, X\right) .
$$

Hence (6.7) becomes

$$
g\left(\nabla_{X} \operatorname{grad} f, Y\right)=-|\mu|^{2} f g(X, Y)+\sum_{i, j=1}^{2} a_{i j} g\left(U_{i}, X\right) g\left(U_{j}, Y\right),
$$

for some functions $a_{i j}$ such that they vanish when the mean curvature vector field is parallel with respect to the normal connection. Thus we have a generalization of the result in $[\mathbf{3}]$ :

THEOREM 6.1. Let $M$ be a submanifold of codimension 2 of a complex submanifold $M^{\prime}$ of a Kähler manifold $\bar{M}$. If $M$ is totally umbilical as a submanifold of $\bar{M}$, the function $f$ satisfies (6.8) for some $a_{i j}, i, j=1,2$, where $a_{i j}$ are such functions that they vanish when the mean curvature vector field is parallel with respect to the normal connection.

\section{References}

[1] S. Kobayashi and K. Nomizu, Foundations of Differential Geometry, Interscience, New York, 1969.

[2] M. Obata, Certain conditions for a Riemannian manifold to be isometric with a sphere, J. Math. Soc. Japan 14 (1962), 333-340. 
[3] M. Okumura, Totally umbilical submanifolds of a Kaehlerian manifold, J. Math. Soc. Japan 19 (1967), 317-327.

[4] M. Okumura and Y. Kubo, A certain function on an even-dimensional real submanifold of a Kaehlerian manifold, Saitama Math. J. 4 (1986), 1-6.

Saitama University

(Received 2506 2003)

255 Shimo-Ookubo

(Revised 1811 2003)

Saitama city

Saitama, Japan

address for correspondence:

5-25-25, Minami Ikuta, Tama-ku,

Kawasaki, Japan

mokumura@h8.dion.ne.jp 